M. Itô

Nagoya Math. J.

Vol. 51 (1973), 45-56

\title{
SUR UNE FAMILLE SOUS-ORDONNÉE AU NOYAU DE CONVOLUTION DE HUNT DONNÉ
}

\author{
dédié à M. le professeur Yukinari Tôki \\ à l'occasion de sa 60ème année
}

\section{MASAYUKI ITÔ}

\section{Introduction}

Soient $X$ un groupe abélien localement compact et dénombrable à l'infini, et $\xi$ sa mesure de Haar. Dans la théorie du potentiel, un noyau de convolution $N$ sur $X$ signifie une mesure de Radon positive dans $X$. Pour une mesure de Radon réelle $\mu$ dans $X, N * \mu$ s'appelle le $N$-potentiel de $\mu$ dès que cette convolution a un sens.

On dit qu'un noyau de convolution $N$ sur $X$ satisfait au principe de domination si, quelles que soient $\varphi, \psi$ de $C_{K}^{+}(X), N * \varphi \leqq N * \psi$ sur $X$ dès que la même inégalité a lieu sur le support de $\varphi$, supp $(\varphi)$. On note ici $C_{K}(X)$ et $C_{K}^{+}(X)$ l'espace vectoriel topologique usuel des fonctions finies et continues dans $X$ à support compact et son cône convexe des fonctions non-négatives, respectivement.

On connaît que, sous une condition additionelle, $N$ satisfait au principe de domination si et seulement si $N$ est un noyau de convolution de Hunt. Un noyau de convolution de Hunt $N$ sur $X$ est, par définition, de la forme $N=\int_{0}^{\infty} \alpha_{t} d t$, où $\left(\alpha_{t}\right)_{t \geqq 0}$ est un semi-groupe vaguement continu de mesures de Radon positives dans $X$; c'est-à-dire, $\alpha_{0}=$ la mesure deDirac $\varepsilon, \alpha_{t} * \alpha_{s}=\alpha_{t+s}$ pour tous $t \geqq 0, s \geqq 0$ et l'application $R^{+}=[0,+\infty) \ni t \rightarrow \alpha_{t}$ est vaguement continue. Dans ce cas, $\left(\alpha_{t}\right)_{t \geqq 0}$ est uniquement déterminé, qui s'appelle le semi-groupe associé au noyau $N$.

On dit qu'une mesure de Radon réelle $\mu$ dans $X$ est bornée si, quelle que soit $\varphi$ de $C_{K}(X), \mu * \varphi$ est bornée sur $X$. On note $M_{b}^{+}\left(R^{+}\right)$la totalité des mesures positives bornées dans la droite réelle $R$ et portées par $R^{+}$.

Received September 29, 1972. 
Pour un noyau de convolution de Hunt $N=\int_{0}^{\infty} \alpha_{t} d t$ sur $X$ et pour une mesure positive $\lambda$ de $M_{b}^{+}\left(R^{+}\right)$, le noyau de convolution

$$
N_{(\lambda)}=\int \alpha_{t} d \lambda(t)
$$

sur $X$ a un sens. On note

$$
D(N ; X)=\left\{N_{(\lambda)} \in D(X) ; \lambda \in M_{b}^{+}\left(R^{+}\right)\right\},
$$

où $D(X)$ est la totalité des noyaux de convolution sur $X$ satisfaisant au principe de domination, et $D(N ; X)$ s'appellera la famille sous-ordonnée au noyau $N$. Cette note sera consacrée à sa caractérisation.

THÉoRÈme. Soit $\lambda$ une mesure positive de $M_{b}^{+}\left(R^{+}\right)$; alors pour que, quels que soient $X$ un groupe abélien localement compact et dénombrable à l'infini et $N$ un noyau de convolution de Hunt sur $X, N_{(\lambda)}$ appartienne à $D(N ; X)$, il faut et il suffit que le noyau de convolution $\lambda$ sut $R$ satisfasse au principe de domination. Dans ce cas, $\lambda$ est un noyau de convolution de Hunt sur $R$ ou 0 .

Dans les articles [2] et [3], on discute que, pour un noyau de convolution de Hunt sur $X$, la somme linéaire de puissances fractionnaires de $N$ satisfait aussi au principe de domination dès que cela a un sens. Mais nous connaissons maintenant que cela est un résultat immédiat de l'énoncé suivant (cf. [4]):

Soit $\left(N_{p}\right)_{p \geqq 0}$ la résolvante associée au noyau $N$; alors, quelle que soit $\lambda(\neq 0)$ une mesure positive sur $R^{+}$de masse totale finie, $\int N_{p} d \lambda(p)$ est aussi un noyau de convolution de Hunt sur $X$.

Si $N=\int_{0}^{\infty} \alpha_{t} d t$, alors

$$
\int N_{p} d \lambda(p)=\iint_{0}^{\infty} \exp (-t p) \alpha_{t} d t d \lambda(p)=\int_{0}^{\infty} \alpha_{t} d t \int \exp (-t p) d \lambda(p) .
$$

En utilisant notre théorème, le présent énoncé déduit immédiatement du principe de domination pour le noyau-fonction

$$
K_{\lambda}(t)= \begin{cases}\int \exp (-t p) d \lambda(p) & t \geqq 0 \\ 0 & t<0\end{cases}
$$

sur $\mathrm{R}$. Cela résulte de la théorie générale, et on peut l'obtenir aussi par le calcul élémentaire (cf. [7]). 
Notre méthode est encore valable pour les noyaux de Hunt (nonconvolution) sur un espace localement compact et dénombrable à l'infini.

\section{La démonstration du théorème}

Commençons d'abord avec quelques lemmes suivants.

LEMME 1. Soient $N=\int_{0}^{\infty} \alpha_{t} d t$ un noyau de convolution de Hunt sur $X$ et $\lambda$ une mesure positive de $M_{b}^{+}\left(R^{+}\right)$; alors le noyau de convolution $N_{(\lambda)}=\int_{0}^{\infty} \alpha_{t} d t$ sur $X$ a un sens.

En effet, quelle que soit $f$ de $C_{K}^{+}(R)$ portée par $R^{+}, f * \lambda$ est bornée sur $R$ et $\operatorname{supp}(f * \lambda) \subset R^{+}$. Donc, quelle que soit $\varphi$ de $C_{K}^{+}(X)$,

$$
\begin{aligned}
+\infty>\int \alpha_{t} * \varphi * \varphi(x)(f * \lambda)(t) d t & =\iint \alpha_{t+s} * \varphi * \varphi(x) f(t) d t d \lambda(s) \\
& =\left(\int \alpha_{t} * \varphi f(t) d t\right) *\left(\int \alpha_{s} * \varphi d \lambda(s)\right)(x)
\end{aligned}
$$

pour tout $x$ de $X$, d'où $N_{(\lambda)}$ a un sens.

LEMME 2. Soit $\lambda$ une mesure positive $(\neq 0)$ de $M_{b}^{+}\left(R^{+}\right)$de masse totale finie; supposons que, quels que soient $X$ un groupe abélien localement compact et dénombrable à l'infini et $N$ un noyau de convolution borné de Hunt sur $X, N_{(\lambda)}$ est aussi un noyau de convolution de Hunt sur $X$. Si $N$ est de la forme

$$
N=\sum_{n=0}^{\infty}(\sigma)^{n},
$$

où $\sigma$ est une mesure de Radon positive dans $X$ de masse totale $\leqq 1$, alors on $a$

$$
N_{(\lambda)}=c \sum_{m=0}^{\infty}\left(\sum_{n=0}^{\infty} a_{n}(\sigma)^{n}\right)^{m}
$$

où cet $a_{n}(n=0,1,2, \cdots)$ sont constantes non-négatives avec $\sum_{n=0}^{\infty} a_{n} \leqq 1$.

On note $(\sigma)^{0}=\varepsilon$, la mesure de Dirac à l'origine, et $(\sigma)^{n}=(\sigma)^{n-1} * \sigma$ $(n \geqq 1)$. Un noyau de convolution $N$ sur $X$ est élémentaire s'il est de la forme $N=c \sum_{n=0}^{\infty}(\sigma)^{n}$, où c et $\sigma$ sont respectivement une constante positive et une mesure de Radon positive dans $X$. Cela est toujours un noyau de convolution de Hunt (cf. [1]). 
Montrons le présent lemme. Soit $Z$ le groupe des entiers; posons $\tilde{N}=\sum_{n=0}^{\infty}\left(\varepsilon_{1}\right)^{n}=\sum_{n=0}^{\infty} \varepsilon_{n}$, où $\varepsilon_{n}$ est la masse d'unité au point $n$. Alors $\tilde{N}$ est un noyau de convolution de Hunt sur $Z$. D'après notre hypothèse, $\tilde{N}_{(2)}$ est aussi un noyau de convolution de Hunt sur $Z$. Evidemment $\tilde{N}_{(\lambda)}(\{0\})>0$, et donc $\tilde{N}_{(\lambda)}$ est élémentaire. On a, d'autre part, $\operatorname{supp}\left(\tilde{N}_{(\lambda)}\right)$ $\subset Z^{+}=\{0,1,2, \cdots\}$, car, quel que soit $t \geqq 0, Z^{+}=\operatorname{supp}(\tilde{N}) \supset \operatorname{supp}\left(\tilde{\alpha}_{t}\right)$, où $\left(\tilde{\alpha}_{t}\right)_{t \geq 0}$ est le semi-groupe associé au noyau $\tilde{N}$. $\tilde{N}$ étant borné, $\tilde{N}_{(\lambda)}$ est aussi borné. Donc il existe constantes non-négatives c et $a_{n}(n=0,1,2, \ldots)$ avec $\sum_{n=0}^{\infty} a_{n} \leqq 1$ telles que

$$
\tilde{N}_{(\lambda)}=c \sum_{m=0}^{\infty}\left(\sum_{n=0}^{\infty} a_{n} \varepsilon_{n}\right)^{m}=c \sum_{m=0}^{\infty}\left(\sum_{n=0}^{\infty} a_{n}\left(\varepsilon_{1}\right)^{n}\right)^{m} .
$$

L'inégalité $\sum_{n=0}^{\infty} a_{n} \leqq 1$ résulte du fait que $\tilde{N}_{(\lambda)}$ est borné (cf. [6]). Soit $N=\sum_{n=0}^{\infty}(\sigma)^{n}$ le noyau de convolution élémentaire dans notre lemme. On peut écrire

$$
N_{(\lambda)}=\sum_{n=0}^{\infty} b_{n}(\sigma)^{n},
$$

où $b_{n}$ est constant $(n=0,1,2, \cdots)$, car

$$
\alpha_{t}=\exp (-t(\varepsilon-\sigma))=\sum_{n=0}^{\infty} \frac{(-t)^{n}(\varepsilon-\sigma)^{n}}{n !} .
$$

On a aussi

$$
\tilde{N}_{(\lambda)}=\sum_{n=0}^{\infty} b_{n}\left(\varepsilon_{1}\right)^{n}=\sum_{n=0}^{\infty} b_{n} \varepsilon_{n} .
$$

En comparant coéfficients, on a

$$
N_{(\lambda)}=c \sum_{m=0}^{\infty}\left(\sum_{n=0}^{\infty} a_{n}(\sigma)^{n}\right)^{m} .
$$

Lemme 3. Soit $\lambda$ la même que ci-dessus et supposons les mêmes conditions que ci-dessus. Alors, quels que soient $X$ un groupe abélien localement compact et dénombrable à l'infini et $N$ un noyau de convolution (non-borné) de Hunt sur $X, N_{(\lambda)}$ satisfait aussi au principe de domination.

En effet, posons $N=\int_{0}^{\infty} \alpha_{t} d t$ et, quel que soit $p>0, N_{p}=\int_{0}^{\infty} \exp (-t p) \alpha_{t} d t$; on a alors 


$$
N+\frac{1}{p} \varepsilon=\frac{1}{p} \sum_{n=0}^{\infty}\left(p N_{p}\right)^{n}
$$

$p N+\varepsilon$ étant aussi un noyau de convolution de Hunt sur $X$, on désigne par $\left(\alpha_{p, t}\right)_{t \geqq 0}$ le semi-groupe associé au noyau $p N+\varepsilon$. On a aussi

$$
\alpha_{p, t}=\sum_{n=0}^{\infty} \frac{(-t)^{n}\left(\varepsilon-p N_{p}\right)^{n}}{n !},
$$

et donc

$$
(p N+\varepsilon)_{(\lambda)}=\sum_{n=0}^{\infty} b_{n}\left(p N_{p}\right)^{n},
$$

où $b_{n}$ est la constante dans le lemme $2(n=0,1,2, \cdots)$. On remarque ici que lorsque l'on écrit

$$
\alpha_{p, t}=\sum_{n=0}^{\infty} f_{n}(t)\left(p N_{p}\right)^{n}
$$

$f_{n}(t) \geqq 0$ sur $R^{+}$pour tout entier $n \geqq 0$ (cf. le noyau de convolution de Hunt $\tilde{N}$ sur $Z)$. Soient c et $a_{n}(n=0,1,2, \cdots)$ aussi les constantes dans le lemme 1. En comparant coéfficients, on obtient que $\sum_{k=0}^{\infty}\left(\sum_{n=0}^{\infty} a_{n}\left(p N_{p}\right)^{n}\right)^{k}$ a un sens et

$$
(p N+\varepsilon)_{(\lambda)} \geqq c \sum_{k=0}^{m}\left(\sum_{n=0}^{\infty} a_{n}\left(p N_{p}\right)^{n}\right)^{k},
$$

d'où $\sum_{m=0}^{\infty}\left(\sum_{n=0}^{\infty} a_{n}\left(p N_{p}\right)^{n}\right)^{m}$ a un sens. En comparant encore coéfficients, on a

$$
(p N+\varepsilon)_{(\lambda)}=c \sum_{m=0}^{\infty}\left(\sum_{n=0}^{\infty}\left(p N_{p}\right)^{n}\right)^{m},
$$

d'où $(N+(1 / p) \varepsilon)_{(\lambda)}$ est un noyau de convolution élémentaire sur $X$. Il est évident que $(N+(1 / p) \varepsilon)_{(\lambda)}$ converge vaguement vers $N_{(\lambda)}$ avec $p \rightarrow+\infty$. Donc, pour le principe de domination pour $N_{(\lambda)}$, il suffit de voir le lemme suivant:

LEMme 4. Soit $\left(N_{m}\right)_{m=1}^{\infty}$ une suite de noyaux de convolution sur $X$ satisfaisant au principe de domination. Si elle converge vaguement vers un noyau de convolution $N$ sur $X$ avec $n \rightarrow+\infty$, alors $N$ satisfait aussi au principe de domination.

Cela est presque connu, et nous faisons seulement quelques remarques. 
Pour le principe de domination pour $N$, il suffit de voir que, quelles que soient $\varphi, \psi$ de $C_{K}^{+}(X)$,

$$
N * \varphi<N * \psi \text { sur } \operatorname{supp}(\varphi) \Rightarrow N * \varphi \leqq N * \psi \text { sur } X
$$

(cf. [6]). D'autre part, la suite $\left(N_{m} * \varphi\right)_{m=1}^{\infty}$ converge uniformément vers $N * \varphi$ sur tout compact dans $X$ avec $m \rightarrow+\infty$.

LEMME 5. Soit $\lambda(\neq 0)$ un noyau de convolution sur $R$ qui appartient $\grave{a} M_{b}^{+}\left(R^{+}\right)$. S'il satisfait au principe de domination, alors $\lambda$ est un noyau de convolution de Hunt sur $R$.

On a, quelle que soit $\varphi$ de $C_{K}(R)$,

$$
\lim _{\substack{x \rightarrow \infty \\ x \in \operatorname{supp}(\lambda)}} \lambda * \varphi(-x)=0 .
$$

D'après le théorème obtenu dans [6], $\lambda$ est un noyau de convolution de Hunt.

Répétons encore notre théorème principal.

THÉOR亡̀me 1. Soit $\lambda$ une mesure positive de $M_{b}^{+}\left(R^{+}\right)$; alors pour que, quels que soient $X$ un groupe abélien localement compact et dénombrable à l'infini et $N$ un noyau de convolution de Hunt sur $X, N_{(\lambda)}$ appartienne à $D(N ; X)$, il faut et il suffit que le noyau de convolution $\lambda$ sur $R$ satisfasse au principe de domination.

Démonstration. Montrons d'abord que la condition est suffisante. Supposons que $\lambda \in M_{b}^{+}\left(R^{+}\right)$satisfait au principe de domination. Alors, d'après le lemme $5, \lambda$ est un noyau de convolution de Hunt sur $R$ ou 0 . Evidemment on peut supposer $\lambda \neq 0$. Soit $N=\int_{0}^{\infty} \alpha_{t} d t$ un noyau de convolution sur $X$. Pour le principe de domination pour $N_{(\lambda)}=\int_{0}^{\infty} \alpha_{t} d \lambda(t)$, d'après le lemme 3 , on peut supposer que $N$ est borné. En considérant la résolvante associée à $N$ et la résolvante associée à $\lambda$, on peut supposer ensuite que $\int d N<+\infty, \int d \lambda<+\infty$ et $\lambda$ est de la forme $\lambda=\sum_{n=0}^{\infty}\left(\lambda_{0}\right)^{n}$, où $\lambda_{0}$ est une mesure positive de $M_{b}^{+}\left(R^{+}\right)$de masse totale $<1$ (cf. le lemme 4). Dans ce cas, on a $\int d \alpha_{t}<1$ pour tout $t>0$. Il existe une fonction définie-négative $\psi$ sur le groupe dual $\hat{X}$ de $X$ avec $\psi(\hat{O})>0$, et une seule telle que, quel que soit $t \geqq 0$,

$$
\hat{\alpha}_{t}=\exp (-t \psi)
$$


où la signe $\wedge$ représente la transformation de Fourier. ${ }^{1)}$ Pour que $N_{(\lambda)}$ soit un noyau de convolution de Hunt sur $X$, il suffit de voir que $1 / \hat{N}_{(\lambda)}$ est définie-négative. On remarque ici $\int d N_{(\lambda)}<\int d \lambda<+\infty$. On a

$$
\begin{aligned}
\frac{1}{\hat{N}_{(\lambda)}} & =\frac{1}{\int \hat{\alpha}_{t} d \lambda(t)}=\frac{1}{\int \exp (-t \psi) d \lambda(t)} \\
& =\frac{1}{\sum_{n=0}^{\infty}\left(\int \exp (-t \psi) d \lambda_{0}(t)\right)^{n}} \\
& =\left(1-\int d \lambda_{0}\right)+\int\left(1-\hat{\alpha}_{t}\right) d \lambda_{0}(t),
\end{aligned}
$$

et par suite $1 / \hat{N}_{(\lambda)}$ est définie-négative. On obtient ainsi que la condition est suffisante.

Réciproquement, on considère le noyau-fonction d'Heaviside $H$ sur $R$; c'est-à-dire, $H(t)=1$ pour $t \geqq 0$ et $H(t)=0$ pour tout $t<0$. Soit $\left(\alpha_{t}\right)_{t \geqq 0}$ le semi-groupe associé à $H$; alors $\alpha_{t}=\varepsilon_{t}$, où $\varepsilon_{t}$ est la mesure de Dirac à $t$. Donc, quel que soit $\lambda$ de $M_{b}^{+}\left(R^{+}\right)$,

$$
\int \alpha_{t} d \lambda(t)=\lambda
$$

On obtient ainsi que la condition est nécessaire. La démonstration est complète.

Remarque. Soient $X$ l'espace euclidien $R^{n}$ à $n(\geqq 1)$ dimensions et $\lambda$ une mesure positive de $M_{b}^{+}\left(R^{+}\right)$. Pour que, quel que soit $N$ un noyau de convolution de Hunt sur $R^{n}, N_{(\lambda)}$ appartienne à $D\left(N ; R^{n}\right)$, il faut et il suffit que $\lambda$ soit un noyau de convolution de Hunt sur $R$ ou 0 .

En effet, dans la présente démonstration, nous utilisons seulement $N=\sum_{n=0}^{\infty} \varepsilon_{n}$ sur $Z$ et le noyau-fonction d'Heaviside sur $R$. Ces deux

1) Une fonction complexe et continue $\psi$ sur $\hat{X}$ est dite définie-négative si l'on a:

(a) $\psi(-\hat{x})=\overline{\psi(\hat{x})}$ pour tout $\hat{x}$ de $\hat{X}$ et $\psi(\hat{O}) \geqq 0$.

(b) ${ }^{\forall} m \in Z^{+},{ }^{\forall} \hat{x}_{0}, \cdots, \hat{x}_{m} \in \hat{X},{ }^{\forall} c_{0}, \cdots, c_{m}$ : nombres complexes tels que $\sum_{i=0}^{m} c_{i}=0$,

$$
\sum_{i=0}^{m} \sum_{j=0}^{m} \psi\left(\hat{x}_{i}-\hat{x}_{j}\right) c_{i} \bar{c}_{j} \leqq 0
$$

On obtient facilement qu'à un semi-groupe vaguement continu $\left(\alpha_{t}\right)_{t \geqq 0}$ avec $\alpha_{0}=\varepsilon$ et $\int d \alpha_{t} \leqq 1$, on peut associer une fonction définie-négative $\psi$ sur $\hat{X}$, et une seule telle que, quel que soit $t \geqq 0, \hat{\alpha}_{t}=\exp (-t \psi)$. 
noyaux peuvent être considérés comme noyaux de convolution de Hunt sur $R^{n}$.

\section{Remarque sur les noyaux de Hunt}

Soient $X$ un espace localement compact et dénombrable à l'infini, et $\xi$ une mesure de Radon positive dans $X$. On note $\mathrm{E}$ et $\mathrm{E}_{0}$ respectivement la $\sigma$-algèbre constituée par tous les ensembles $\xi$-mesurables dans $X$ et sa sous-famille des ensembles relativement compacts. Dans l'article [5], on a introduit la notion de noyau $N$ relatif à $X$ et à $\xi$ et discuté les potentiels par rapport au noyau $N$ et la composition de deux noyaux. Voir [5] pour les définitions énoncées ci-dessous. On note, pour deux noyaux $N_{1}, N_{2}$ relatifs a $X$ et à $\xi, N_{1} N_{2}$ le noyau composé de $N_{1}$ et $N_{2}$. Le noyau d'unité relatif à $X$ et à $\xi$ s'écrit $U$.

On dit qu'une famille $\left(N_{t}\right)_{t \geqq 0}$ de noyaux relatifs à $X$ et à $\xi$ s'appelle un semi-groupe continu si l'on a:

(1) $N_{t} N_{s}=N_{t+s} \quad{ }^{\forall} t \geqq 0, \quad{ }^{\forall} s \geqq 0$.

(2) $N_{0}=U$.

(3) L'application $t \rightarrow N_{t}$ est fortement continue ; c'est-à-dire, quelle que soit $f$ de $B_{K}(X ; \xi)$, l'application $t \rightarrow N_{t} f$ est continue pour la topologie forte de $L_{1 \mathrm{loc}}(X ; \xi)$.

On note ici $B_{K}(X ; \xi)$ et $B_{K}^{+}(X ; \xi)$ l'ensemble des fonctions $\xi$-mesurables et bornées dans $X$ à support compact et son cône convexe des fonctions non-négatives, respectivement.

Un noyau de Hunt $V$ relatif à $X$ et à $\xi$ est, par définition, un noyau relatif à $X$ et à $\xi$ de la forme $V=\int_{0}^{\infty} N_{t} d t$, où $\left(N_{t}\right)_{t \geqq 0}$ est le semi-groupe continu de noyaux relatifs à $X$ et à $\xi$. De la même manière que dans le cas où $N$ est un noyau de Hunt continu (cf. [1]), $\left(N_{t}\right)_{t \geqq 0}$ est uniquement déterminé pour $N$. On dit aussi que $\left(N_{t}\right)_{t \geqq 0}$ est le semi-groupe associé au noyau $N$. Posons, pour $p \geqq 0, V_{p}=\int_{0}^{\infty} \exp (-t p) N_{t} d t$; alors

$$
(p-q) V_{p} V_{4}+V_{p}-V_{q}=0 \quad\left({ }^{\forall} p \geqq 0,{ }^{\forall} q>0\right)
$$

et $V_{p}$ converge fortement vers $V$ avec $p \rightarrow 0$. Donc si un noyau de Hunt $V$ relatif à $X$ et à $\xi$ vérifie la condition (*); quel que soit $e$ de $\mathrm{E}$ avec $\xi(e)>0, V(e, X)>0$ et $V(X, e)>0$, alors $V$ satisfait au principe de domination (cf. [5]). Au lieu du lemme 4, on a le lemme suivant:

LEMME 6. Soit $\left(V_{n}\right)_{n=1}^{\infty}$ une suite de noyaux relatifs à $X$ et à $\xi$ satis- 
faisant au principe de domination. Si elle converge fortement vers un noyau $V$ relatif $\grave{a} X$ et $\grave{a} \xi$ avec $n \rightarrow+\infty$ et si $V$ vérifie la condition (*), alors $V$ satisfait au principe de domination.

D'après la condition $(*)$ pour $V$, il suffit de voir que, quelles que soient $f, g$ de $B_{K}^{+}(X ; \xi)$,

$$
V f<V g \text { s-p.p. } \operatorname{sur}\{x \in X ; f(x)>0\} \Rightarrow V f \leqq V g \xi \text {-p.p. sur } X
$$

(cf. [5]). La notation $\xi$-p.p. signifie "presque partout pour $\xi "$ ". Cette implication déduit de la même manière usuelle (cf. [5]).

Par conséquent, on obtient le théorème suivant:

THÉORÈme 2. Soient $V=\int_{0}^{\infty} N_{t} d t$ un noyau de Hunt relatif $\grave{a} X$ et $\grave{a}$ $\xi$, et $\lambda$ un noyau de convolution de Hunt appartenant $\grave{a} M_{b}^{+}\left(R^{+}\right)$. Alors $V_{(\lambda)}=\int N_{t} d \lambda(t)$ satisfait au principe de domination dès que cela est un noyau relatif $\grave{a} X$ et $\grave{a} \xi$.

LEMME 7. Soient $\left(N_{t}\right)_{t \geqq 0}$ un semi-groupe continu de noyaux relatifs $\grave{a} X$ et $\xi$, et $\lambda$ un noyau de convolution de Hunt appartenant $\grave{a} M_{b}^{+}\left(R^{+}\right)$. Alors $V_{(\lambda)}=\int N_{t} d \lambda(t)$ vérifie la condition (*) dès que $V_{(\lambda)}$ a un sens.

En effet, supposons qu'il existe un ensemble $e$ de $\mathrm{E}$ avec $\xi(e)>0$ tel que $V_{(2)}(X, e)=0$. Dans ce cas, on peut supposer $e \in \mathbf{E}_{0}$. On a, quel que soit $K$ un compact de $X, N_{t}(K, e)=0$ pour tout $t$ de $\operatorname{supp}(\lambda)$, car l'application $t \rightarrow N_{t}(K, e)$ est continue. D'après $\operatorname{supp}(\lambda) \ni 0, U(K, e)=0$, d'où $U(X, e)=0$, d'où contradiction. De la même manière on a, quel que soit $e$ de $\mathrm{E}$ avec $\xi(e)>0, N(e, X)>0$.

Démonstration du théorème 2. D'après le lemme 6, on peut supposer $\int d \lambda<+\infty$ (cf. la démonstration du théorème 1) et encore que $\lambda$ est une fonction continue et bornée dans $(0,+\infty)$, car si l'on peut écrire $\lambda=\sum_{n=0}^{\infty}\left(\lambda_{0}\right)^{n}$, où $\lambda_{0}$ est une mesure positive de $M_{b}^{+}\left(R^{+}\right)$avec $\int d \lambda_{0}<1$, alors, quelle que soit $\varphi$ de $C_{K}^{+}(R)$ portée par $R^{+}$et avec $\int \varphi(t) d t \leqq 1$, $\sum_{n=0}^{\infty}(\lambda * \varphi)^{n}$ est continue et bornée dans $(0,+\infty)$.

Posons aussi $V_{p}=\int_{0}^{\infty} \exp (-t p) N_{t} d t$ pour tout $p>0$; alors cela est un noyau relatif à $X$ et à $\xi$, et on a 


$$
V+\frac{1}{p} U=\frac{1}{p} \sum_{n=0}^{\infty}\left(p V_{p}\right)^{n}
$$

où $\left(p N_{p}\right)^{0}=U$. Posons ensuite

$$
N_{p, t}=\sum_{n=0}^{\infty} \frac{(-t p)^{n}\left(U-p V_{p}\right)^{n}}{n !}\left({ }^{\forall} t>0\right) \text { et } \quad N_{p, 0}=U \text {; }
$$

alors $N_{p, t}$ est un noyau relatif à $X$ et à $\xi$ et $\left(N_{p, t}\right)_{t \geqq 0}$ est un semi-groupe continu de noyaux relatifs à $X$ et à $\xi$. On a

$$
V+\frac{1}{p} U=\int_{0}^{\infty} N_{p, t} d t
$$

D'après la remarque ci-dessus pour $\lambda,(V+(1 / p) U)_{(\lambda)}$ est un noyau relatif à $X$ et à $\xi$. D'après le théorème 1 et en utilisant la même manière que dans le lemme 3 , $(V+(1 / p) U)_{(\lambda)}$ est élémentaire, d'où il satisfait au principe de domination. Il est facile de voir que, quel que soit $t \geqq 0$, $N_{p, t}$ converge fortement vers $N_{t}$ avec $p \rightarrow+\infty$, et donc, d'après le théorème de Lebesgue, $(V+(1 / p) U)_{(\lambda)}$ converge fortement vers $N_{(\lambda)}$ avec $p \rightarrow+\infty$. En vertu des lemmes 6 et $7, V_{(\lambda)}$ satisfait au principe de domination. La démonstration est ainsi complète.

\section{Appendice}

Dans la présente discution, le principe de domination pour les noyax de convolution appartenant à $M_{b}^{+}\left(R^{+}\right)$est essentiel. On se propose de fournir une condition explicite.

Proposition. Soit $\kappa$ un noyau de convolution sur $R$ appartenant à $M_{b}^{+}\left(R^{+}\right)$et de la forme $d \kappa=k(t) d t$, où $k$ est une fonction $>0$, finie et continue sur $R^{+}$et $k=0$ dans $R-R^{+}$. Si, quel que soit $a \geqq 0, k(t) / k(t+a)$ est décroissante (au sens large) sur $R^{+}$, alors $\kappa$ satisfait au principe de domination.

Démonstration. Il suffit de voir que, quels que soient $r_{0}, r^{\prime}$ avec $0<r_{0}<r^{\prime}$, il existe une mesure de Radon positive $\varepsilon_{r_{0}, r^{\prime}}^{\prime}$ dans $R$ portée par $\left[r_{0}, r^{\prime}\right]$ telle que $\kappa \geqq \kappa * \varepsilon_{r_{0}, r^{\prime}}^{\prime}$ dans $R$ et $\kappa=\kappa * \varepsilon_{r_{0}, r^{\prime}}^{\prime}$ au sens des mesures dans $\left(r_{0}, r^{\prime}\right)$. Soit $n$ un entier $>0$. D'après notre hypothèse,

$$
k(t) \geqq \frac{k\left(r_{0}\right)}{k(0)} k\left(t-r_{0}\right)
$$


dans $\left[r_{0},+\infty\right)$. Posons $a_{0}=k\left(r_{0}\right) / k(0)$. S'il existe un point de $\left(r_{0}, r^{\prime}\right)$ où la fonction $(1-1 / n) k(t)-a_{0} k\left(t-r_{0}\right)$ prend la valeur positive, on pose

$$
r_{1}=\inf \left\{t \in\left(r_{0}, r^{\prime}\right) ;\left(1-\frac{1}{n}\right) k(t)>a_{0} k\left(t-r_{0}\right)\right\}
$$

et $a_{1}=1 / n\left(k\left(r_{1}\right) / k(0)\right)$. S'il existe encore un point de $\left(r_{1}, r^{\prime}\right)$ où la fonction $(1-1 / n) k(t)-a_{0} k\left(t-r_{0}\right)-a_{1} k\left(t-r_{1}\right)$ prend la valeur positive, on détermine $r_{2}$ et $a_{2}$ de la même manière. Par récurrence, il est facile de voir qu'il existe un entier $m_{n}>0$, une famille $\left(r_{m}\right)_{m=0}^{m_{n}}$ de nombres avec $r_{0}<r_{1}<\ldots<r_{m_{n}} \leqq r^{\prime}$ et une famille $\left(a_{m}\right)_{m=0}^{m_{n}}$ de nombres $>0$ telles que

$$
\begin{aligned}
\sum_{m=0}^{m_{n}} a_{m} k\left(t-r_{m}\right) & \leqq k(t) \text { dans } R \text { et }\left(1-\frac{1}{n}\right) k(t) \leqq \sum_{m=0}^{m_{n}} a_{m} k\left(t-r_{m}\right) \\
& \leqq k(t) \text { sur }\left[r_{0}, r^{\prime}\right] .
\end{aligned}
$$

En posant $\mu_{n}=\sum_{m=0}^{m_{n}} a_{m} \varepsilon_{r_{m}}$, la suite $\left(\mu_{n}\right)_{n=1}^{\infty}$ est vaguement bornée. Un point vaguement adhérent $\varepsilon_{r_{0}, r^{\prime}}^{\prime}$ de $\left(\mu_{n}\right)_{n=1}^{\infty}$ est une mesure que nous avons désiré. La démonstration est complète.

Corollaire. Soit $\lambda$ une mesure positive dans $R^{+}$. Si un noyau de convolution $\kappa$ sur $R$ est de la forme

$$
d \lambda= \begin{cases}\left(\int \exp (-t s) d \lambda(s)\right) d t & \text { sur } R^{+} \\ 0 & \text { dans } R-R^{+},\end{cases}
$$

alors $\kappa$ satisfait au principe de domination.

Cela résulte immédiatement de la présente proposition et du calcul élémentaire.

\section{Bibliographie}

[1] J. Deny: Noyaux de convolution de Hunt et noyaux associés à une famille fondamentale, Ann. Inst. Fourier (Grenoble), 12, 1962, p. 643-667.

[ 2 ] F. Hirsch: Sur une généralisation d'un théorème de M. Itô, C. R. Acad. Sc. Paris, 271, 1970, p. 1236-1238.

[ 3 ] M. Itô: Sur la somme des noyaux de Dirichlet, ibid., 271, 1970, p. 937-940.

[4] - Remarque sur la somme des résolvantes, Proc. Japan Acad., 46, 1970, p. 243-245.

[5] — : Sur le principe divers du maximum et le type positif, Nagoya Math. J., 44, 1971, p. 133-164.

[6] — : Une caractérisation du principe de domination pour les noyaux de convolution, à paraître. 
[7] M. Kishi: An example of a non-symmetric convolution kernel satisfying the domination principle, Nagoya Math. J., 48, 1972, p. 189-196.

Université de Nagoya 